\title{
UNITÉ PROPOSÉE BASÉE SUR LES ACTIVITÉS LUDIQUES POUR DÉVELOPPER QUELQUES COMPÉTENCES DE LA COMMUNICATION ORALE EN FRANÇAIS CHEZ LES ÉLÈVES DU CYCLE PRIMAIRE AUX ÉCOLES DISTINGUÉES DE LANGUES Lamyaa Mohamed El Sayed El Awady
}

\begin{abstract}
This research aimed to verify the effectiveness of edutainment activities to develop some skills of oral communication in French. To achieve this goal, the researcher developed three tools: a questionnaire designed to determine the oral communication skills needed by the students in the research sample, a test and an assessment grid of the oral communication skills. The research sample included a group of 30 students in grade five at the distinguished language schools at Sinbellawin in the Governorate of Al - Dakahlia. This sample studied the proposed unit according to the play activities. The results of this research have shown that there is an effectiveness of the proposed unit based on edutainment activities to develop the skills of oral communication among students from the fifth grade to the language schools.
\end{abstract}

Key words: edutainment activities, oral communication, primary cycle, distinguished language schools.

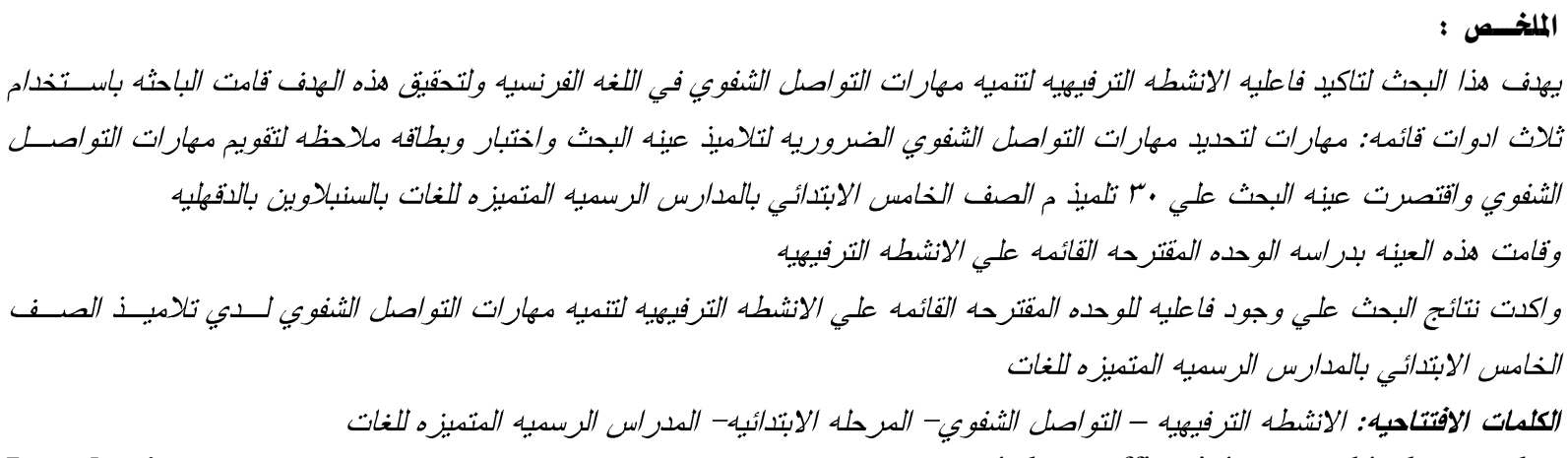

Introduction

La langue est un don de Dieu à l'homme pour se distinguer d'autres êtres vivants, aussi c'est le premier moyen pour penser et interagir avec les autres. Le besoin de communiquer entre les êtres vivants entraîne le développement du langage sous différents aspects. En outre, la langue française, comme l'une des langues étrangères, comporte quatre compétences essentielles: lire, écrire, écouter, parler. La communication orale donne l'occasion aux apprenants d'apprendre une langue étrangère et de leur permettre de communiquer correctement. La vie est la communication. Pas de vie personnelle ou professionnelle sans paroles ni échanges. La communication orale permet de partager, et peut améliorer la lecture et l'écriture.

Malgre l'importance de la communication orale pour l'apprentissage d'une langue étrangère, surtout la langue francaise, il y a une faiblesse dans le niveau des élèves aux compétences de la communication orale, notamment aux différents cycles éducatifs d'après les études suivantes : (Mohamed 2006), (El- Sayed 2006), (Abou Elftouh 2013), (El-Alawy 2016). Par conséquent, les stratégies, les méthodes et les activités d'apprentissage qui aident à développer les compétences en communication orale devraient être utilisées en classe de FLE. Les activités ludiques ont prouvé leur efficacité pour développer les compétences de communication orale. Il y a plusieurs études qui ont démontré l'efficacité des activités ludiques en vue de développer ces compétences comme: (Achouri 2007) (Magly 2008), (Guerni 2011), (Osterlund 2011), (Fievet 2011), (Limousin 2012), (Deveyneix, Guibert et Colas 2013), (Leleu 2014).

Dhif (2012) souligne que de tout temps, pédagogues et psychologues se sont intéressés à la part de l'émotion et du plaisir dans l'apprentissage. Or, il s'avère que les activités ludiques présenteraient la situation la plus authentique d'utilisation de la langue dans la salle de classe et elles permettraient aux apprenants de franchir un pas dans le processus d'apprentissage.

De plus, Hamza (2014) souligne que l'activité ludique comme un moyen d'aider les élèves à construire progressivement du système de vocabulaire, structuré et cohérent. Le jeu ludique, conduit de façon méthodique, pourrait développer chez l'élève la capacité à s'approprier les mots et leur étymologie et maîtriser peu à peu l'usage.

Problématique de la recherche

La problématique de la recherche rérside en la faiblesse chez les élèves de la cinquième année primaire aux écoles officielles distinguées de 
langues en communication orale. La chercheuse s'est assurée de ce problème comme suit:

1- La chercheuse a assisté à un certain nombre des cours avec des enseignants au cycle primaire aux écoles distinguées de langue et a observé que la communication orale ne prend pas le soin suffisant en comparaison avec l'expression écrite.

2- Les orientateurs de la langue française au cycle primaire ont confirmé le manque des méthodes et des stratégies d'apprentissage modernes et appropriées à développer les compétences en communication orale.

3- La chercheuse a appliqué un test exploatif à 30 élèves en cinquième année primaire aux écoles officielles distinguées de langues à Sinbellawien au Gouvernorate d'Al - Dakahlia pour vérifier leur niveau dans les compétences de la communication orale en français. On a obtenu les résultats suivants:

1- Le pourcentage de la moyenne des notes concernant la compétence (Ajuster sa voix pendant la communication orale) est $41 \%$.

2- Le pourcentage de la moyenne des notes concernant la compétence (Prouduire un vocabulaire riche et varié) est $40 \%$.

3- Le pourcentage de la moyenne des notes concernant la compétence (Parler avec confiance sans confusion) est $38 \%$.

4- $\quad$ Le pourcentage de la moyenne des notes concernant la compétence (Respecter les règles grammaticales lors de la communication orale) est $45 \%$.

5- Le pourcentage de la moyenne des notes concernant la compétence (écouter en vue de bien distinguer les mots) est $39 \%$.

6- Le pourcentage de la moyenne des notes concernant la compétence (Donner des réponses correspondant à des questions lors de la situation de communication orale) est $43 \%$.

7- Le pourcentage de la moyenne des notes concernant la compétence (Comprendre le sens des mots inconnus à travers le sens général de la phrase) est $37 \%$.

Ces résultats montrent la faiblesse des élèves de la cinquième année primaire dans les compétences de communication orale. Pour faire face à ce problème, cette recherche a pour but de répondre aux questions suivantes:

1- Quelles sont les compétences de la communication orale nécessaires aux élèves de la cinquième année primaire aux écoles officielles distinguées de langues?

2- Jusque' à quel niveau les élèves de l'échantillon de la recherche maîtrisent - ils ces compétences?
3- Quelle est l'efficacité de l'unité proposée basée sur les activités ludiques pour déveloper les compétences de la communication orale chez les élèves de la cinquième année primaire?

Objectifs de la recherche

1-Déterminer les compétences de la communication orale nécessaires aux élèves de la cinquième année primaire.

2- Mesurer l'efficacité de l'unité proposée basée sur des activités ludiques pour développer la communication orale chez l'échantillon de la recherche.

Importance de la recherche

Cette recherche essaye de/d':

1- Encourager les élèves à participer activement au processus d'apprentissage pour améliorer leur performance orale.

2- Inciter les chercheurs en didactique du FLE à prendre soin de la faiblesse de la communication orale et développer cette compétence.

3- Attirer l'attention des orienteurs et des enseignants de français à l'utilisation des activités ludiques en classe aux différents cycles éducatifs.

\section{Hypothèses de la recherche}

1- Il existe une différence statistiquement significative au niveau (0.05) entre les moyennes des notes des élèves du groupe expérimental au pré/ post test des compétences de la communication orale en faveur du post test.

2- Il existe une différence statistiquement significative auprès des élèves du groupe expérimental à la grille d'évaluation des compétences de la communication orale et au niveau supposé de maîtrise $(70 \%)$ en faveur de la post application de la grille d'évaluation.

3- Il existe une efficacité de l'unitée proposée basée sur les activités ludiques pour développer les compétences de la communication orale chez les élèves de l'échantillon de la recherche.

Limites de la recherche

L'échantillon de la recherche se détermine en un seul groupe expérimental composé de 30 élèves en cinquième année primaire aux écoles officielles distinguées de langues à Sinbellawien au Gouvernorat d'Al-Dakahlia qui étudient le français comme deuxième langue étrangère.

\section{Outils de la recherche}

1- Un questionnaire des compétences da la communication orale.

2- Un test des compétences de la communication orale.

3- Une grille d'évaluation des compétences de la communication orale.

Méthodologie de la recherche

La chercheuse a eu recours à deux méthodes: 
A- La méthode descriptive : En ce qui concerne les principes philosophiques de la recherche (les activités ludiques et la communication orale).

B- La methode quasi - expérimentale: En ce qui concerne l'étude expérimentale et l'application $\mathrm{du}$ test et de la grille d'évaluation afin de mesurer l'efficacité de l'unité proposée basée sur les activités ludiques pour développer la communication orale chez les élèves en cinquième année primaire aux écoles officielles distinguées de langues.

Démarche de la recherche

1- Pour répondre à la première question, on a procédé comme suit:

1- Passer en revue les ouvrages et les études antérieures concernant la communication orale pour en tirer profit pour l'élaboration du questionnaire des compétences de la communication orale.

2- Élaborer un questionnaire des compétence de la communication orale nécessaires aux élèves de la cinquième année primaire aux écoles distinguées de langues.

3- Présenter ce questionnaire à un jury specialiste en FLE afin de déterminer les compétences les plus nécessaires et convenables à l'échantillon de la recherche.

2- Pour réprondre à la deuxième question, on a procéde comme suit:

1- Élaborer un pré / post test et une grille d'évaluation des compétences de la communication orale pour détecter à quel niveau les élèves de la cinquième année primaire aux écoles officielles distinguées de langues maîtrisent ces compétences.

2- Présenter ces deux outils aux members du jury pour déterminer leur validité.

3- Pour répondre à la troisième question, on a procédé comme suit:

1- Élaborer une unité proposée basée sur les activités ludiques.

2- Présenter cette unité aux members du jury afin de déterminer sa validité.

3- Choisir les élèves du groupe expérimental de la recherche.

4- Appliquer le test avant l'enseignement de l'unité proposée sur l'échantillon de la recherche.

5- Enseigner l'unité proposée à l'échantillon de la recherche selon les activités ludiques.

6- Appliquer le test aprés l'enseignement de l'unité proposée sur l'échantillon de la recherche.

7- Analyser les résultats en employant les méthodes statistiques convenables.

8- Discuter et interpréter les résultats.

9- Proposer des recommandations et des suggestions.
Terminologie de la recherche

- Les activités ludiques

De Grandmont (1997) définit les activités ludiques comme "une action librement consentie avec un début et une fin, le désir et la volonté de se concentrer sur un problème afin de le résoudre".

Cuq (2003) définit les activités ludiques comme "une activité d'apprentissage qui est guidée par des règles de jeu et pratiquée pour le plaisir qu'elle procure".

- La définition opérationnelle: la chercheuse définit les activités ludiques comme une activité d'apprentissage simple, amusante et passionnante de la vie réelle qui aide l'apprenant à s'exprimer, à prendre décision, à faire des choix et à développer ainsi son autonomie.

- La communication orale

Selon Moisan (2000) la communication orale est " un outil qui favorise la construction de la pensée et qui permet la communication de cette pensée une fois construite".

Quatrevaux (2002) définit la communication orale comme "une capacité à utiliser la parole pour exprimer ou échanger des idées ou des informations en milieu de travail ou dans la vie de tous les jours".

- La définition opérationnelle: la chercheuse définit la communication orale comme un acte qui permet à l'apprenent d'exprimer oralement ses idées, ses sentiments, ses aspirations, de partager ses connaissances et de communiquer avec les autres.

Principes philosophiques de la recherche

A- Les activités ludiques

évolution du concept « jeu / ludique »

Thirion (2016) assure que la sorphologie ludique a été crée par Claudia Sanchez et Ricardo Lopez en 1985. Elle est née dans le besoin d'application de la sophrologie d'une facon facile, agréable et compréhensible par le jeu dans le champ social et prévenitif. Elle s'adresse au plus grand nombre, des enfants, des jeunes, des adultes, des personne âgées, dans les écoles, les entreprises et les institution.

Elle offre au développement personnel une manière ludique pédagogique de découverte de soi, de développement et de changement accessible, à tout public, peu importe son âge, son sexe, sa profession ou sa différence culturelle et ethnique, le langage de la vie et de la créative dépasse les frontières et rassemble les gens.

Principes de didactisation des activités ludiques Jiménez (2017) assure que les jeux et activités de communication dans la classe de langue sont au moins trois types d'informations pour les enseignants:

- Les objectifs linguistiques ou une activité pragmatique. 
- Le matériel: supports de texts, audio, visuels, instructions,

- Les actions : préparation, déroulement et les changements possibles.

En revanche, il est plus peu de trouver dans ce type d'ouvrages à visée pédagogique des indications sur d'autres composantes importantes telles que: ( $\mathrm{La}$ dimension cognitive- La dimension socioaffective- La rétro-information- L'activité métalinguistique- L'itérativité).

Importance des activités ludiques en classe de FLE

Amel (2015) souligne que le ludique, dans l'enseignement/apprentissage, permet aux apprenants d'utiliser de façon collaborative et créative l'ensemble de leurs ressources verbales et communicatives. Cette nouvelle approche innovatrice, dont découlerait une nouvelle transmission du savoir, trouverait toute sa plénitude dans le plaisir de l'action et du jeu. Elle rendrait l'école plus agréable et plus attrayante aux yeux de l'élève en lui procurant bonheur et bien être. Dans ce contexte, Kervran (1996) assure que les activites ludiques aident à la participation effective de l'apprenant dans l'apprentissage. Des diverses activités sont proposées à l'apprenant, le faisant intervenir dans le cadre d'un ou plusieurs objectifs d'apprentissage précis: il s'agit par exemple de faire un exercice, de répondre à une question fermée ou ouverte, de dessiner, de découvrir un contenu, etc. Le jeu est considéré comme une activité de communication qui est fondée sur le défi. C'est par une bonne maîtrise de la langue que l'enfant va pouvoir entrer efficacement dans l'activité.

Caractéristiques de la forme ludique

Selon Ameur (2015) le jeu, comme l'une des formes d'activités ludiques, possède plusieurs caractéristiques:

1- L'exploration: Jouer, c'est explorer le monde, se mesurer à lui, rassembler ses propres forces pour résoudre une difficulté, vaincre un obstacle. Le jeu permet à l'enfant l'exploration du monde extérieur en même temps qu'il s'explore lui-même, c'est-à-dire, il découvre le monde autour de lui et se présente par l'action du jeu.

2- La socialisation : Le jeu permet à l'enfant joueur de communiquer avec les autres (adversaires ou partenaires). En élaborant des échanges avec eux à travers le jeu, il fait sa personnalité, donc il se socialise.

3- La compétition : Le jeu a un objectif et constitue un enjeu. La compétition est donc à propos de soi, des choses ou des autres. Le jeu signifie l'acceptation directe de l'épreuve, et avec le but de réussir. Si l'enfant réussisse ou échoue, il doit garder l'espoir dans un autre succès.
4- La règle : Le jeu suit des règles limites qui aident l'enfant à apprendre, à respecter et à se faire respecter.

Conditions de l'utilisation des activités ludiques en classe du FLE.

Ourida (2012) assure qu'un jeu bien utilisé en classe de langue a autant de pertinence que d'autres types d'activités ludiques. Afin d'utiliser convenablement cet outil pédagogique, il convient de s'interroger sur:

- L'objectif: Quel est le but visé du jeu choisi? Pour que ce dernier ait de sens et d'intérêt.

- Le public: 'A quel public s'adresse le jeu? Il sera sélectionné en fonction du niveau des apprenants.

-Les connaissances: Quelles sont les connaissances que l'apprenant doit avoir acquis pour pratiquer le jeu choisi ?

- L'intérêt: Quel est l'intérêt que présente le jeu, sur ce qu'il apporte dans le cadre de la séquence?

- Le moment: Ầ quel moment de la séquence fautil employer le jeu? Est-ce au début du cours? Estce à la fin du cours?

Types des activités ludiques

Les activités ludiques sont un moyen pédagogique qui facilite l'apprentissage du FLE, et motivent les apprenants pour aimer cette langue étrangère. Le jeu est l'une de ces activités: (Irani 2001), (De Greave 2006), (Taghezout 2009), (Harkou 2015) A- Jeux de créativité:

Ils engagent une réflexion plus personnelle, orale ou écrite, de la part de l'apprenant et demandent plus de créativité et d'imagination, l'accent est mis sur les capacités linguistiques du participant qui est invité à produire des formes originale, insolites, voire même poétiques.

B- Jeux culturels:

Ils se réfèrent plus à la culture et aux connaissances des élèves.

\section{C- Jeux dérivés du théâtre:}

Ils changent la salle de classe en scène de théâtre, les élèves en acteurs, et qui reposent sur l'improvisation la dramatisation, les jeux de rôle, les jeux de simulation font partie de ce groupe et il va de soi que la simulation globale est l'activité la plus complète.

\section{D- Jeux de communication}

Les élèves ont besoin de communiquer et utiliser des compétences linguistiques dans une situation ludique. Grâce à cette activité, les enfants apprennent progressivement à s'exprimer leur compréhension et de se faire comprendre. Ces jeux incluent au moins deux joueurs, tels que :

- Parler à un correspondant au téléphone: donner réponses correctes à des questions et savoir comment poser une question.

- Jouer le postier et prendre l'information sur un message, dramatiser une histoire, jouer par téléphone sans fil et chasse au trésor. Il s'agit 
d'un outil pédagogique adaptable à des situations variées de classe en réponse à différents objectifs. E- Jeux linguistiques:

Ils incluent les jeux de grammaire, de morphologie ou syntaxiques, lexicaux, phonétiques et orthographiques qui permettent de gérer certaines règles de la langue ou de découvrir sa structure et ses caractéristiques ou la mémorisation de ses règles de fonctionnement.

\section{F- Jeux ludiques}

Le jeu ludique connu comme une activité libre par excellence (n'a pas des règles, pas obligatoire, ni esthétisme, il est l'acte du plaisir instinctif du joueur). En plus, c'est une activité physique ou mentale, qui s'y livre d'autre but que le plaisir qu'elle procure, aussi le jeu ludique est une façon d'explorer et de découvrir les connaissances à travers l'action ludique du jeu.

\section{G- Jeux éducatifs}

Ce terme est mystérieux parce qu'on pourrait se demander s'il est avant tout ludique ou simplement un exercice à but éducatif? En fait, que le mystère du terme jeu éducatif lui serait constitutive. Les enseignants devraient fournir aux élèves des exercices tout en regardant le jeu, leur permettraient d'atteindre le but éducatif qu'ils ont fixé. Cela signifie que multiusagers, et de changer les règles de jeu éducatif lorsque son fonctionnement a été saisi par l'élève.

\section{H- Jeux pédagogiques}

Le jeu pédagogique est soumis à un système de règles qui doit être respecté, il présente d'autres objectifs plus exigeantes pour déterminer sans perdre le personnage principal plaisant et agréable, son objectif principal est de transférer ou d'évaluer l'appropriation des savoirs et des comportements linguistiques, c'est-à-dire les apprentissages. Ceci est le deuxième jeu se classe dans une autre optique qui est purement didactique (jeu éducatif ou pédagogique).

\section{Aavntages de l'activité ludique}

Cuq et Gruca (2003) montrent que d'une part, les activités ludiques rendent le cours moins astreignant, instaurent des relations les plus communs dans les interactions, améliorent les échanges verbaux entre les apprenants et permettent particulièrement de dépasser la relation traditionnelle maître/élèves. D'autre part, les activités ludiques font une certaine socialisation possible et permettent à l'élève de se familiariser avec les autres acteurs dans son apprentissage : les co-apprenants et l'enseignant. De même, les jeux pourraient contribuer à l'apprentissage la "coconstruction des connaissances" (entre coapprenants). De plus, l'activité ludique peut être un assistant efficace dans l'acquisition du langue, car les compétences linguistiques minimales permettent déjà de créer des combinaisons infinies et d'explorer systématiquement les possibilités de la langue. De l'autre part, la plupart des activités ludiques ne nécessitent aucun matériel et peuvent connaître des variantes pour mieux répondre aux objectifs ou au niveau des apprenants.

Activités ludiques et la communication orale Selon Sauvé et Kaufman (2010) le jeu est l'activité de communication basée sur le défi pour réaliser un ensemble exact par respecter des règles bien définies. Il fait le besoin et le désire de connaitre la langue : pour une participation efficace et pour gagner, il doit écouter, comprendre et parler. Les acquis linguistiques sont fixés ou modifiés dans un environnement motivant et naturel. Par conséquent, il serait impossible de ne pas travailler sur le langage oral ou de l'écriture parce que la fonction du langage est de pouvoir communiquer: il doit être capable de comprendre et d'être compris. Le jeu pourrait être une excellente façon pour y parvenir. Pour lier les mots avec des actions et des gestes correspondants et permet de rappeler les concepts étudiés.

\section{B- La communication orale}

Importance de la communication orale

Germain et Netten (2005) soulignent qu'une langue est d'abord une habileté utilisée pour transmettre des messages authentiques, oralemet et par l'écrit. Cependant, il ne fait aucun doute qu'il est facile qu'apprendre de communiquer oralement en langue seconde ou étrangère et permet à l'élève de faire des conversations avec des locuteurs natifs et ainsi, d'arriver à une autre culture, on ne semble pas se rendre compte, parfois, de tout l'endroit. L'interaction entre enseignant et l'élève ou entre les élèves dans les activités de groupes offre de nombreuses possibilités d'utiliser la langue apprise en contexte originale. Pendant l'activité d'interaction, l'élève aura alors tendance à mettre l'accent sur le message qu'il veut transmettre, ce qui aidera à rendre de plus en plus "automatiques" les structures de la langue et les vocabulaires utilisés.

Collège de bois- de Boulogne (2009) montre que la communication orale permet de rappeler les idées, les faits, les événements, etc. Elle est une partie des relations entre différents individus et peuples. La communication orale nécessite une excellente préparation qui contrôle certain aspects, mais aussi une certaine aptitude pour improviser. La nécessité de communiquer entre les êtres vivants de même espèce et d'espèces différentes entraîne le développement du langage dans les différents aspects. La communication orale est donc une façon privilégiée qui aide à partager les idées, les preoccupations et les orientations, etc.

\section{Facteurs influançant la communication orale}

Desmons et al (2005) soulignent que pour parler de système oral, il est nécessaire de prendre en compte un grand nombre de facteurs issus tant du discours émis que de la situation de communication dans laquelle il est émis. Ces facteurs, qui jouent un rôle 
principal dans l'intercompréhension, peuvent être classés dans trois catégories :

1. Les traits de l'oralité : Ces traits oraux ont différentes fonctions grammaticales et sémantiques :

- Les prosodiques (les arrêts, les accents d'insistance, les modifications de courbe intonative, le débit).

- Les contractions : les professeurs de français font attention à enseigner la grammaire correctement. Cependant, dans une interview avec des natifs, les raccourcis sont communs «y a pas », « chai pas », « t'as vu » etc.

- Les hésitations et les pauses.

- Les parasitages en conversation.

2. Le jeu social : Il rassemble les accents régionaux et sociaux, les registres linguistiques et les implications culturelles.

3. Le corps : Le geste, l'imitation et la proxémie, en d'autres termes, la distance entre les personnes et les contacts physiques parmi les locuteurs jouent un rôle principal dans la communication orale. En fait, apprendre et communiquer dans la langue étrangère consiste à écouter et à parler.

Toute action de travail oral conduit à prendre conscience de considérer les deux côtés de l'oral en même temps: la compréhension et l'expression.

\section{Volets de la communication orale}

La communication orale se divise en trois volets: Saskatchewan (2000), Chatha (2007), Benamar (2009)

\section{1- L'écoute}

L'étudiant est un récepteur actif ou un destinataire qui est responsable de reconstruire le sens du message. Cette reconstruction de la signification se produit dans un contexte particulier et est dirigée par l'intention de communication de l'élève. La réponse de l'étudiant au message est d'une importance primordiale. L'écoute comprend l'ouïe, la réflexion et la pensée critique. Elle développe avec l'âge et la maturité intellectuelle. Écouter comprend trois actions: (Obtenir physiquement le message - Faire attention aux idées présentées successivement dans un message - Donner un sens global d'un message). En outre, la pratique d'écoute exige que l'étudiant ait à sa disposition les façons pour réaliser son projet d'écoute et qu'ils peut les utiliser efficacement. Ces façons sont les stratégies de planification, de gestion de son projet d'écoute. L'écoute se compose de trois étapes essentielles la préécoute, l'écoute et la post écoute. Dans ces trois étapes, l'élève est en étroite interaction avec le contexte et le discours qu'il entend ou visionne, pour reconstruire leur signification.

\section{2- L'interaction}

L'interaction dans la communication orale est très importante, c'est un échange dynamique dans lequel les personnes expriment spontanément leurs besoins, leurs réactions, leurs idées et leurs points du vue. Bien que interprétant les messages de leurs interlocuteurs et interlocutrices. Elle comporte trois étapes: La préinteraction, l'interaction et la postinteraction.

\section{3- L'exposé oral}

L'exposé oral est une intervention orale adressée à un auditoire (déjà connu ou qu'on prétend connaître en cours d'exposé) rassemblé pour l'écouter afin de lui transmettre des informations, lui décrire ou lui expliquer quelque chose. Cette expérience demande une bonne préparation qui doit porter tout autant sur le contenu et la structure, sur une prise en considération du public, de ses valeurs, de ses intérêts et qui permettra à l'exposant d'oraganiser son discours de façon logique et lui servira d'aide mémoire lors de sa présentation. L' exposé oral a son importance dans le développement de la confiance en soi et dans la libération de la parole des élèves. Par conséquent, il est une forme de communication orale qui nécessite un grand souci de la langue et de la structure d'un message. L'apprenant construit la signification de son message. Il contient trois étapes: Le préexposé, l'éxposé et le postéxposé. Dans chacune de ces trois étapes, l'étudiant est en interaction constante avec le discours et le contexte. Il est soit dans le cas de la reception, soit dans le cas d'émission pour la compréhension ou de se faire comprendre. En plus, l'étudiant peut faire un retour sur ses progrès, et sur ses apprentissages. Alors, il existe un lien langagier de l'interconnexion entre ses trois sous-domaines car l'état d'écoute signifie nécessairement la situation d'expression orale. C'est la relation dynamique entre les différents domaines de la langue qui permettra aux étudiants de communiquer efficacement dans différents contextes de la vie quotidienne scolaire et sociale et l'utilisation de la langue française afin de penser, apprendre, se construire une identité et se créer une atmosphère culturelle. En outre, les volets et les processus de communication orale montrent que la compétence communicative n'est pas posée comme simple connaissance, mais aussi comme disposition à agir et à s'engager dans une performance communicative.

\section{Fonctions de la communication orale}

Mélanie (2011) détermine sept fonctions de la communication orale:

1- La fonction instrumentale : cela permet d'obtenir quelque chose quotidienne, de prendre un rendez-vous.

2- La fonction de contrôle : son rôle vient quand les interlocuteurs tentent de changer le comportement de l'autre (appel à l'aide, choix de discours électoral, signalisation routière...).: Communicateurs tente de modifier le 
comportement de l'autre (l'appel à l'aide, le choix du discours et la signalisation routière ).

3- La fonction d'information : intervient dans un contexte éducatif, mais aussi dans les relations interpersonnelles. Les communicateurs échangent de clarifier des incertitudes, la résolution des conflits......

4- La fonction d'expression d'une réalité intérieure : s'accomplit lorsque les interlocuteurs expriment leur état émotionnel des conditions somatiques ou dans un contexte social spécifique (condoléances, félicitations...). Dans ce cas la communication non-verbale est généralement très importante.

5- La fonction de contact social et de stimulation : est mise en jeu quand les individus communiquent pour le plaisir pour interagir avec les autres et évitent ainsi l'isolement et la solitude. La communication est alors décrite comme une série de stimuli physiologiques essentiels (visuelles, auditives, tactiles...).

6- La fonction d'allègement de l'anxiété : est proche de la précédente, communiquer aide les personnes à trouver un soulagement dans les échanges interpersonnels.

7- La fonction liée au rôle social : est présente quand les individus communiquent dans le travail par exemple. La communication avec le public sera différente en fonction de rôle social des interlocuteurs.

Modes de la communication orale

Marc et Picard (2008) distinguent deux modes de communication chez l'homme: La communication, cela correspond à ce qui est dit par les mots, et la connexion analogique, qui serait plus difficile à identifier car elle est liée à des aspects non-verbaux des échanges. Il y a aussi une distinction entre:

- Une communication verbale, suivie d'aspects phonologiques, lexicaux, sémantiques, syntaxiques et pratiques.

- Une communication non-verbale, Ceci est démontré par les regards, la gestualité, les mimiques et postures, ainsi que par la proxémique et les modulations vocales. Les significations non verbales expriment et personnifient les propos du locuteur. Les éléments mimo-gestuels formés par le contexte extra-linguistique de l'échange. La prosodie, la voix et le rythme définissent, quant à eux, le contexte paralinguistique. Ces deux contextes interagissent avec le contexte linguistique du locuteur et sont d'importance similaire aux propos langagiers puisqu'ils influencent la signification du message.

\section{évaluation de la communication orale}

Lafontaine et Messier (2009) démontrent qu'il y a plusieurs outils pour l'évaluation orale, y compris la grille d'évaluation, qui est un outil de mesure de prendre en compte les spécificités de toute action, d'un produit ou un procédé en fournissant une liste d'observations et une méthode d'enregistrement des observations. Cela peut concerner un étudiant ou un groupe d'étudiants et elle peut être remplie par les étudiants ou par l'enseignant. Il existe aussi une évaluation par les pairs qui rend les étudiants actifs et les amène pour prendre en charge la communication orale en identifiant les forces et les faiblesses de leurs pairs. Cette façon d'évaluation a besoin d'évaluer la quantité, la valeur, la qualité et le succès des productions ou de réussite de l'apprentissage de leurs pairs.

étude éxpérimentale de la recherche

I- Choix de l'échantillon de la recherche:

L'échantillon de la recherche se compose d'un groupe expérimental 30 élèves en cinquième année primaire aux écoles distinguées de langues qui a appris selon les activités ludiques. La chercheuse a choisi cet échantillon qui étudie le français comme deuxième langue étrangère pour les raisons suivantes:

1- On a appliqué une étude explorative sur un échantillon de 30 élèves en cinqième année primaire aux écoles distinguées de langues à $\mathrm{Al}$ Senbelawin- au Gouvenorat d'Al Dakahlia pour vérifier leur niveau dans la communication orale. Cette étude a montré que les élèves sont faibles en communication orale.

2- Les orinteurs et les enseignants de français au cycle primaire ont montré le niveau faible des élèves en compétences de la communication orale et leurs points du vue insistent à la nécessite de les développer.

3- Ces élèves ont des savoirs précédents en français car ils ont déjà étudié le français pendant la quatrième année primaire. Ces savoirs leur ont permis d'acquérir des nouvelles connaissances, de bien comprendre et d'accomplir les activités ludiques concernant la communication orale.

II- Préparation des outils de la recherche

1- Un questionnaire des compétences de la communication orale nécessaires aux élèves de la cinquième année pimaire.

2- Un test des compétences de la communication orale.

3- Une grille d'évaluation des compétences de la communication orale.

1-1- Objectifs du questionnaire

Afin de préparer le questionnaire, on a d'abord défini son objectif qui est la détermination des compétences de la communication orale nécessaires aux élèves de la cinquième année année primaire aux écoles distinguées de langues afin d'utiliser la langue française d'une manière compréhensible et d'apprendre la prononciation claire et effective.

1-2- Description du questionnaire

Le questionnaire comprend quatre pages: 
a- La première page contient le titre de la recherche et ses objectifs.

b- Les trois autres pages contiennent trente_six compétences de la communication orale en français nécessaires aux élèves du cycle primaire aux écoles distinguées de langues.

1-3- Déroulement du questionnaire

Pour vérifier la validité du questionnaire, la chercheuse l'a soumis au jury qui est composé de:

a- Certains spécialistes en langue et en didactique du F.L.E.

b- Certains orienteurs et enseignants de la langue française.

La chercheuse leur a demandé de donner leurs points de vue en ce qui concerne:

1- L'adéquation des compétences au niveau des élèves de la cinquième année primaire.

2- La suppression des compétences non nécessaires.

3- L'addition des compétences non -inclues.

4- La modification des compétences si nécessaire.

2- Test des compétences de la communication orale

\section{2-1- Objectifs du test:}

1- Évaluer quelques compétences de la communication orale en français chez les élèves la cinquième année primaire aux écoles distinguées de langues.

2- Mesurer l'efficacité des activités ludiques pour développer quelques compétences de la communication orale en français chez les élèves de l'échantillon de la recherche.

\section{2-3- Description du test}

Le test se compose de six pages:

1- La première page contient le titre de la recherche, les objectifs et les consignes du test.

2- Les cinq autres pages sont consacrées à neuf questions. Celles-ci mesurent neuf compétences de la communication orale:

a) La première et la huitième question mesure les compétences du vocabulaire.

b) La deuxième question mesure les compétences de la compréhension.

c) La troisième question mesure les compétences d'écoute.

d) La quatrième question mesure les compétences de la fluidité.

e) La cinquième question mesure les compétences de la grammaire.

f) La sixième et la septième question mesurent les compétences de la prononciation.

g) La neuvième question mesure les compétences du contenu.(Annexe II)

\section{2-4- étude pilote}

La chercheuse a appliqué une étude pilote un test pour vérifier sa fidélité,sa validité et déterminer sa durée. Pour réaliser ces objectifs, la chercheuse a appliqué ce test sur un échantillon différent de l'échantillon de la recherche. Celui-ci est composé de 30 étudiants de la cinquième année primaire.

\section{2-4-1- Durée du test}

On a calculé la durée de l'application du test par la formule suivante:

Durée

Temps consommé par chaque élève pour tous les membres du groupe

$$
\text { Durée }=\frac{2700}{30}=90 \text { minutes. }
$$

\section{2-4-2- Fidélité du test}

Pour vérifier la fidélité du test, on a réappliqué le même test dans une période de 4 semaines sur le même échantillon en calculant le coefficient de corrélation des notes des élèves dans les deux applications par la formule de Pearson.On a comparé les résultats de deux tests dans les deux fois:

$\mathrm{R}=\frac{N \Sigma X Y-\Sigma X \sum Y}{\sqrt{\left[\mathrm{N} \Sigma X^{2}-(\Sigma X)^{2}\right]\left[\mathrm{N} \Sigma Y^{2}-(\Sigma Y)^{2}\right]}}$

$\mathrm{R}=$ Le coefficient de corrélation

$\mathrm{N}=$ Le nombre des élèves

$\sum=$ La somme

$\mathrm{X}=$ Les notes des élèves dans la première application

$\mathrm{Y}=$ Les notes des élèves dans la deuxième application

$\mathrm{X} 2=$ Les carrés des notes des élèves dans la première application

Y2= Les carrés des notes des élèves dans la deuxième application

$\mathrm{R}=0.79$

Alors, le coefficient de fidélité $=0.79$

Cette valeur indique que le test est fidèle.

\section{2-4-3- Validité du test:}

Pour vérifier la validité du test:

1- On a présenté le test à un jury composé de certains spécialistes en langue et en didactique du F.L.E et certains orienteurs et enseignants de français. Ils ont décidé que le test est valide.

2- On a calculé la validité à partir de sa fidélité en appliquant la formule suivante:

La validité $=\sqrt{ }$ La fidélité

La validité $=\sqrt{ } 0.79=0.88$

Cette valeur est élevée. Donc, le test est valide.

3- Grille d'évaluation de la communication orale

\section{3-1- Objectifs de la grille}

Cette grille vise à estimer et à mesurer le niveau de maîtrise des compétences de la communication orale chez les élèves en cinqième année primaire aux écoles distinguées de langues.

3-2- Description de la grille d'évaluation de la communication orale.

La grille d'évaluation de la communication orale comprend quatre pages:

a- La première page contient le titre de la recherche et les objectifs de la grille. 
b- Les trois autres pages contiennent sept items qui visent à évaluer les compétences de la communication orale chez l'echantillon de la recherche.

3-3- Validité de la grille d'évaluation de la communication orale

Après avoir construit les items de la grille, on l'a présentée sous une forme préliminaire aux membres du jury. On leur a demandé de donner leurs points de vue en ce qui concerne:

1- La clarté et la justesse de la langue de la grille.

2- Les items de la grille sont valides à mesurer les objectifs visés.

3- L'adéquation de son contenu par rapport aux objectifs visés.

- Contenu de l'unité proposée

-L'unité se compose de cinq leçons: quatre leçons d'enseignement et une leçon de révision, incluses activités ludiques proposées pour le développement de la communication orale, y compris les compétences individuelles et collectives.
- Ensiegnement de l'unité proposée d'aprés les activités ludiques

Aprè avoir appliqué le pré-test, on a commencé l'enseignement de l'unité proposée au deuxième semestre depuis 15 mars jusqu' à 19 avril de l'année scolaire 2016 / 2017. L'expérimentation a duré six semaines à raison de deux cours (période) par semaine, (Période d'une heure et demi).

Résultats de la recherche

I- Analyse statistique des résultats

- Première hypothèse:

- Il existe une différence statistiquement significative au niveau (0.05) entre les moyennes des notes des élèves du groupe expérimental au pré/ post test des compétences de la communication orale en faveur du post test.

Pour vérifier cette hypothèse, on a calculé les moyennes des notes des élèves au pré/ post test en ce qui concerne les compétences de la communication orale en utilisant le test $(\mathrm{T})$. Le tableau suivant indique ces résultats:

Tableau N0.1

Résultats du groupe expérimental au post- test

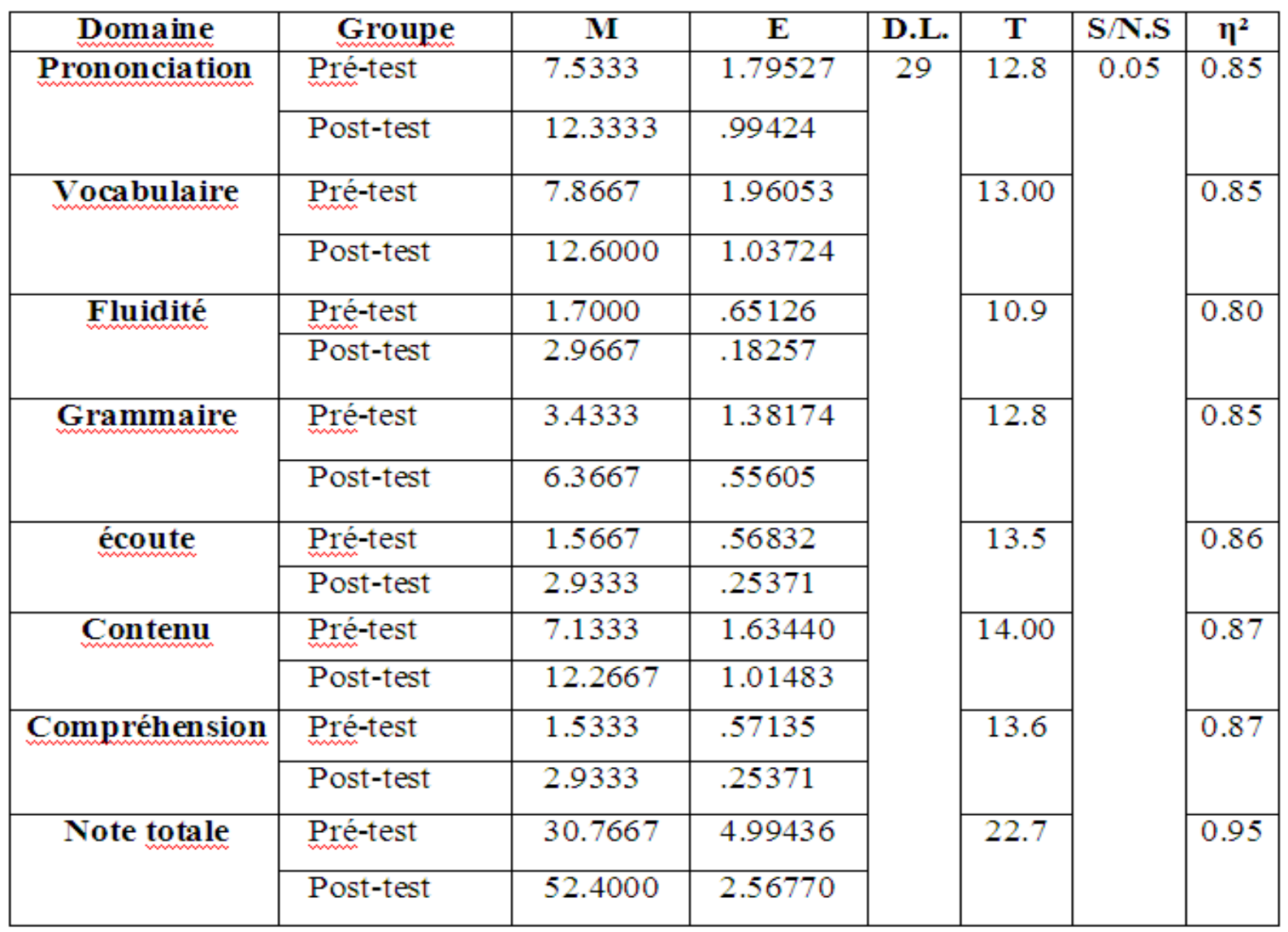

M : La moyenne des notes.

E. : écart - type.

D.L. : Degré de liberté.

T. : Valeur de « $\mathrm{T} »$ test.

S. : Signification.

ç 2: Eta Carré. 
Ce tableau indique que les valeurs de (t) aux domaines sont entre $(10.9-14)$ et à la note totale (22.7). Ces valeurs sont significatives au niveau (0.05). Donc, il y a une différence statistiquement significative entre la moyenne des notes des élèves du groupe expérimental au post- test des compétences de la communication orale en faveur du post test. Par conséquent, la première hypothèse a été vérifiée.

- Deuxième hypothèse

Il existe une différence statistiquement significative auprès des élèves du groupe expérimental à la grille d'évaluation des compétences de la communication orale et au niveau supposé de maîtrise $(70 \%)$ en faveur de la post applicaion de la grille d'évaluation.

Pour vérifier cette hypothèse, la chercheuse a utilisé le test " $\mathrm{T}$ " pour montrer la différence entre la moyenne des notes des élèves du groupe expérimental à la grille d'évaluation des compétences de la communication orale et au niveau de maîtrise $(70 \%)$. Ce tableau montre les résultats suivants:

Tableau N0.2

Résultats du groupe expérimental à la grille d'évaluation

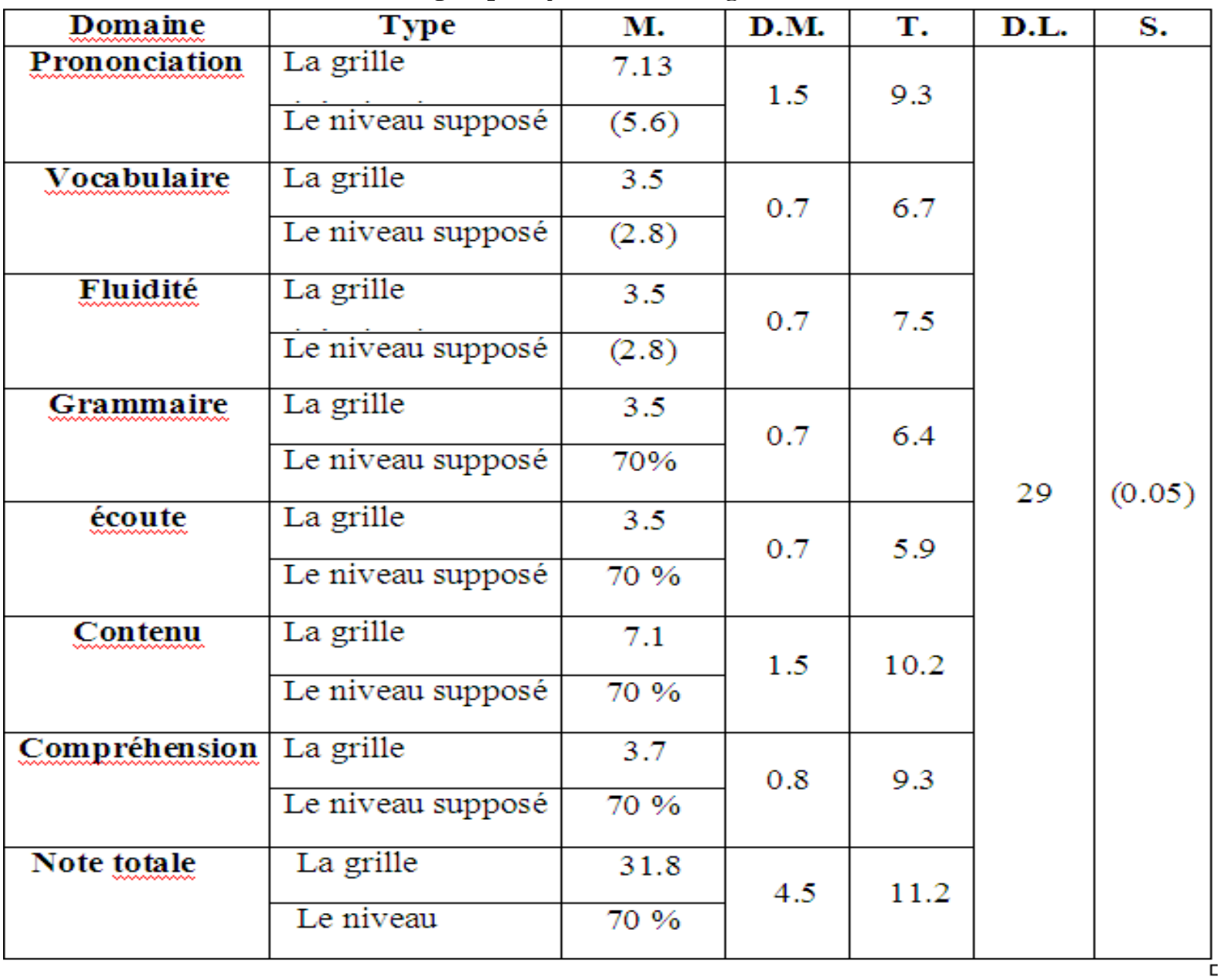

Ce tableau indique que la valeur des moyennes des notes du groupe expérimental à la post application de la grille d'évaluation (31.8) est plus élevée que celle du niveau supposé de maîtrise $70 \%$ (27.3) de la note totale de la grille d'évaluation. Ainsi, il y a une différence statistiquement significative des élèves du groupe expérimental sur la grille d'évaluation des compétences de la communication orale et au niveau supposé de maîtrise (70\%) en faveur de la grille d'évaluation. Par conséquent, la deuxième hypothèse a été vérifiée.

Troisième hypothèse

Il y a une efficacité de l'unité proposée basée sur les activités ludiques pour développer les compétences en communication orale chez les élèves de l'échantillon de la recherche. Pour vérifier cette hypothèse trois, on a calculé la proporation du gain modifié de Black et la proporation d'efficacité de McGogian. Le tableau suivant montre ces résultats: 
Tableau No.3

Propotion du gain modifié de Black et propotion d'efficacité de McGogian.

\begin{tabular}{|c|c|c|c|c|}
\hline N.T. & M.N.Pré & M.N.Post. & P.G.M. & P.E. \\
\hline 60 & 30.8 & 52.4 & 1.2 & 0.74 \\
\hline
\end{tabular}

Ce tableau indique que la proportion du gain modifié de Black est (1.2) et la proportion d'efficacité de McGogian est (0.74). Ces valeurs indiquent aussi qu'il y a une efficacité de l'unite proposée basé sur les activités ludiques pour développer des compétences en communication orale chez les élèves de l'échantillon de cette recherche. Par conséquent, la troisième hypothèse a été vérifiée.

\section{II- Interprétation des résultats}

Selon les résultats de la recherche qui ont été soulevés, on peut conclure que les activités ludiques sont efficaces pour développer les compétences de la communication orale chez l'échantillon de la recherche. Cela s'explique par les facteurs suivants:

1- Les activités ludiques ont permis aux élèves d'utiliser de façon nouvelle et personnelle, le vocabulaire et les structures acquises au cours des leçons en les faisant sortir du cadre dans lequel ils ont appris.

2- Elles leur ont permis d'utiliser toutes leurs ressources de communication de manière individuelle, collaborative et créative.

3- Elles ont aidé à découler une nouvelle transmission des connaissances acquises chez les élèves en leur faisant jouer pleinement à l'action de plaisir et de divertissement.

4- Elles ont permis à rendre l'école plus agréable et attrayante aux yeux des élèves en leur fournissant le bonheur et bien être.

5- Elles ont incité les élèves à s'exprimer oralement avec plaisir en les aident à la mémorisation des mots, ce qui a leur permis d'avoir un vocabulaire très riche.

6- Elles ont développé les connaissances des élèves et la capacité d'ajouter plus d'amusement et de divertissement.

7- Elles ont donné aux élèves l'occasion de communiquer oralement les uns avec les autres dans la classe.

8- Elles ont stimulé la curiosité, l'enthousiasme et la motivation d'apprentissage chez les élèves.

9- Elles ont permis de connaître les choses en jouant avec d'autres enfants de son âge pour découvrir leurs différences, et ressentir les réactions des autres.

10- Elles ont aidé à créer une atmosphère favorable et agréable de plaisir d'apprentissage dans la classe.

11- Grâce à ces activiés ludiques, les élèves ont réinvesti leur pré requis en évoquant des métiers déjà rencontrés en classe.
12- L'association image/mot a encouragé les élèves à se rappeler des sens des mots et c'est pourquoi ils ont su employer les mots de manière correcte dans les activités d'apprentissage d'évaluation.

\section{III- Recommandations de la recherche}

D'après les résultats de la recherche, les recommandations suivantes ont été formulées:

1- élaborer des activités ludiques pour favoriser les compétences de la communication orale en FLE chez les apprenants dans chaque cycle d'enseignement.

2- Les enseignants doivent prendre les activités ludiques comme method adaptée à l'enseignement de la communication orale en français dans la classe.

3- Pour améliorer les compétences de la communication orale en français, les élèves doivent pratiquer ces compétences et les mettre en pratique à plusieurs fois sous la supervision de l'enseignant.

4- Inciter les apprenants à interagir efficacement en utilisant des nouvelles méthodes comme les activites ludiques.

5- Encourager les élèves à communiquer oralement dans ou dehors l'école. Cela les aide à développer leurs compétences en communication et une attitude positive envers l'oral.

6- Donner la priorité à l'oral et particulièrement à la communication orale dans le processus d'apprentissage/enseignement de la langue francaise.

7- Constituer les programmes du cycle primaire afin de pratiquer la langue comme une langue de communication en classe du FLE.

\section{IV-Suggestions de la recherche}

Comme le montrent les résultats de cette recherche, on présente quelques sujets de recherche:

1- Mesurer l'efficacité d'utilisation des activités ludiques pour acquérir des compétences de l'expression écrite en français chez les étudiants du cycle secondaire.

2- Élaborer un programme proposée basé sur le portfolio électronique pour développer les compétences en communication orale chez les étudiants du cycle universitaire.

3- Vérifier l'efficacité d'utilisation des activités ludiques pour développer les compétences de la comprehension en lecture en français chez les élèves du cycle primaire. 
4- Mesurer l'efficacité des activités ludiques en vue d'acquérir les règles grammaticales chez les élèves du cycle primaire.

5- Faire une étude comparative entre les activités ludiques et l'apprentissage hypride pour développer les compétences de la compréhension orale chez les élèves du cycle préparatoire.

6- élaborer un programme proposée basé sur hypertextualité a fin de développer les compétences en communication orale chez les étudiants /enseignants.

7- élaborer unité proposée basée sur les activités ludiques en vue de développer le vocabulaire en français chez les élèves $\mathrm{du}$ cycle préparatoire.

Bibliographie

a- Références en langue française

Abou Elftouh, H-S. (2013) : Utilisation de

l'approche interculturelle pour déveloper les compétences de la communication orale chez les élèves de cycle préparatoire, Thèse de Magistère non publiée, Faculté de Pédagogie, Université d'Ain- Chams.

- Achouri, S. (2007): Compétences langagières et activités ludiques en classe de FLE, Thèse de Magistère, Faculté des Lettres et des Sciences Humaines, Université Colonel Hadj LakhdarBatna. $\quad$ http://eprints.univbatna2.dz/492/1/le_Achouri\%20Souad.pdf

- Amel, M. (2015): Le ludique comme stratégie d'enseignement/apprentissage en classe de langue, Université d'Oran 2. file:///C:/Users/B7B6 1/AppData/Local/Temp/0b 88eb0a58f675986b2b3a2bb60aa000.pdf

- Ameur, M. (2015) : Le jeu de l'oie comme activité de motivation à l'expression orale et l'enrichissement du vocabulaire dans une classe du FLE. Cas des élèves de la $5^{\text {ème }}$ année primaire, Thèse de Magistère, Université de M'Sila.

http://revuestaps.univmsila.dz/facultell/images/fll_d oc/documents/memoire/fr/master/2015/ameur\%20 meriem\%20master\%20msila\%202015.pdf

- Benamar, R. (2009): Stratégies d'aide à la production orale en classe de FLE, Algérie, Université de Tlemcen, $\mathrm{N}^{\circ} 8$.

- Chatha, H. (2007): Le rôle de l'exposé orale dans le développement des compétences communicatives orales, Thèse de Magistère, Faculté des Lettres et des Langues, Université de Mentouri Constantine, Algérie. http://bu.umc.edu.dz/theses/francais/CHT1085.pd $\underline{\mathrm{f}}$

- Collège de bois- de Boulogne (2009): La communication orale. http://nbeauchemin.profweb.ca/H09/docs/ORAT OIRE.pdf
- Cuq, J.P. (2003) : Dictionnaire de didactique du français langue étrangère et seconde, Paris: CLÉ International.

- Cuq, J. P. \& Gruca, I. (2003): Cours de didactique de français langue étrangère et seconde, Grenoble: Presse Université de Grenoble,

- De Grandmont, N. (1997): Pédagogie du jeu, jouer pour apprendre, Paris: De Boeck.

- De Greave, S. (2006) : Apprendre par les jeux, Paris: De Boeck.

- Desmons, F. et al. (2005) : Enseigner le FLE. Pratiques de Classe, Paris: Belin.

- Deveyneix, I. Guibert, A. \& Colas, G. (2013): Stratégies ludiques pour favoriser la prise de parole, Espace Pédagogique de L'académie de Poitiers, No. 185.

- Dhif, A. (2012): Compétences communicatives et simulation globale en classe de FLE, Cas de la 2ème année secondaire, Thèse de Magistère, Faculté des Lettres et des Langues, Universite de Mohamed Kheider Biskra.http://dspace.univbiskra.dz:8080/jspui/bitst ream/123456789/5129/1/sf133.pdf

- El-Alawy, N. (2016) : Utilistion d'une stratégie actionnelle proposée pour développer les compétences de la communication orale des futurs ensignants de FLE à la Faculté de Pédagogie, Thése de Magistère non publiée, Faculté de Pédagogie, Université de Damietta.

- El- Sayed, R. (2006): Un programme proposé pour déveloper les compétences de la communicatuins orale chez les étudiants de la 4 ème année au départment de français à la Faculté de pédagogie, Thèse de Magistère non publiée, Faculté de Pédagogie, Université d'Assiut.

- Fievet, F. (2011) : Des jeux ludiques et interactifs pour parler l'anglais.

http://www.speakyplanet.fr/blog/jeux-ludiquesparler-anglais/

- Germain, C. \& Netten, J. (2005): Place et rôle de l'oral dans l'enseignement/apprentissage d'une L2, Babylonia, Vol 8, No2.

- Guerni, S. (2011): L'amélioration de la prise de parole à travers les activités ludiques, Thèse de Magistère, Faculté des Lettres et des Langues, Université de Mohamed Kheider -Biskra. http://dspace.univbiskra.dz:8080/jspui/bitstream/1 23456789/5093/1/sf108.pdf

- Hamza, S. (2014): L'activite ludique comme outil pédagogique favorisant l'acquisition du vocabulaire dans une classe de FLE. Cas des apprenents de 5ème année primaire, Thèse de Magistère, Faculté des Lettres et des langues, Université de Mohamed Khider-Bisakra. http://dspace.univbiskra.dz:8080/jspui/bitstream/1 23456789/5765/1/HAMZA\%20Sara.pdf 
- Harkou, L. (2015): Pour l'utilisation de l'activité ludique en Algérie dans la classe de FLE, Gerflint, Algérie. No 22.

- Irani, F. (2001): Créativité et aspect ludique dans l'enseignement du français (contexte indien): analyse d'un essai, France: Presses Universitaires du Septentrion.

- Jiménez, F. (2017): Principes de didactisation des activités ludiques dans les pratiques orales, Cahiers de l'Apliut, Universitaire de Technologie, France, Vol.36, No2.

- Kervan, M. (1996) : L'apprentissage actif de l'anglais à l'école, Armand: Colin.

- Lafontaine, L. \& Messier, G. (2009): Représentations de l'enseignement et de l'évaluation de l'oral chez des enseignants et des élèves du secondaire en français langue d'enseignement, Nouvel Ontario, Québec, N³4.

- Limousin, L. (2012): Comment favoriser la prise de parole en classe de langues?. http://dumas.ccsd.cnrs.fr/dumas00736084/document

- Marc, E. \& Picard, D. (2008) : Relations et communications interpersonnelles,Belgique: Dunod.

- Mélanie, R. (2011): Élaboration d'outils de communication destinés à des patients traumatisés crannies graves pris en charge en service de soins de suite et de réadaptation, Thèse de Magistère, Université de Bordeaux, Segalen.

https://dumas.ccsd.cnrs.fr/dumas01307120/document

- Mohamed, N. (2006): L'effet d'un programme proposé basé sur le jeu de rôle dans l'enseigement de la langue française pour développer quelques compétences de la communication orale chez les étudiants de première année à la Faculté de Pédagoeie de Minia, Thèse de Magistère non publiée, Faculté de Pédagoeie, Université de Minia.

- Moisan, M.(2000): Valoriser la discussion pour reviver la communication orale.Québec Français, No 118.

- Ourida, H. (2012): Les activités ludiques illustrées pour une perspective interculturelle du vocabulaire, Thèse de Magistère, Université de Mohamed Khider-Biskra, Algérie.

http://dspace.univbiskra.dz:8080/jspui/bitstream/1 23456789/5123/1/sf129.pdf
- Quatrevaux, A. (2002) : Journaux de formation, analyse de disours et communication orale, Paris: L'Harmattan.

- Sauvé, L \& Kaufman, D. (2010): Jeux et simulations éducatifs: Études de cas et leçons apprises, Québec: Presses de l'Université du Québec.

- Saskatchewan, R. (2000) : Français programme d'études niveau élémentaire écoles Fransaskoises, Programme d'études, Ministère de l'Éducation de la Saskatchewan, Bureau de la Minorité de Langue Officielle, Canada.

https://www.k12.gov.sk.ca/docs/francais/fransk/fr an/elem/copy.html

- Taghezout, A. ( 2009) : L'activité ludique dans l'apprentissage des mots en français langue étrangère,Thèse de Magistère, Université d'Oran, Algérie. oran1.dz/document/TH3524.pdf

- Thirion, Ch. (2016): Sophrologie et activités ludiques pour les 3-12 ans, Paris: Mango Éditions.

b- Reférences en langue anglais:

- Leleu, S. (2014): French speaking activites (KS2): Fun ways to get KS2 pupils to talk to each other in french, A Brilliant Education ,Vol.35.

- Osterlund, C. (2011): Fun with speaking. file:///C:/Users/B7B6 1/AppData/Local/Temp/Pu blicSpeakingActivities.pdf

C- Reférences en langue arabe

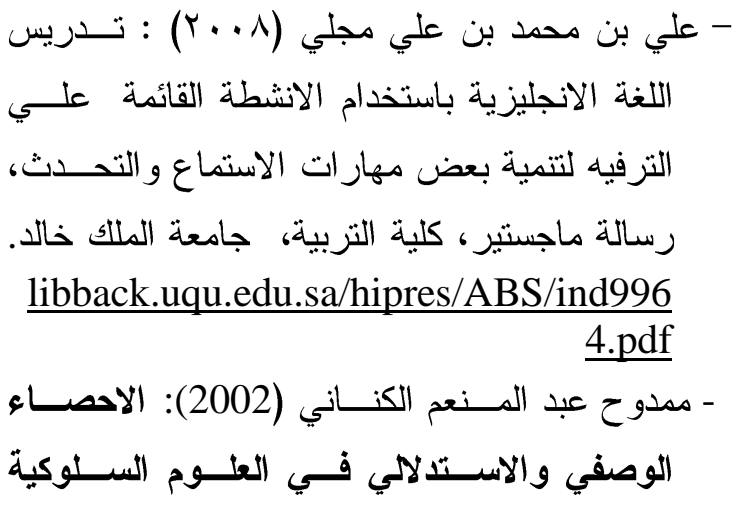

والاجتماعية، دار النشر للجامعات، الطبعة الثانية. 